\title{
(6) OPEN ACCESS \\ Antithrombotic therapy in atrial fibrillation: aspirin is rarely the right choice
}

\section{'Rayne Institute, St. Thomas' Hospital, London, UK ${ }^{2}$ Physiological Laboratory, University of Cambridge, Cambridge, UK}

\section{Correspondence to}

Dr lan N Sabir, Physiological Laboratory, University of Cambridge, Downing Street, Cambridge CB2 3EG, UK; ins20@cam.ac.uk

Received 9 August 2012 Revised 8 November 2012 Accepted 20 January 2013 Published Online First 12 February 2013

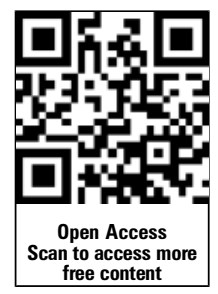

To cite: Sabir IN, Matthews GDK, Huang CL-H. Postgrad Med J 2013;89:346-351.

\author{
Ian N Sabir, ${ }^{1,2}$ Gareth D K Matthews, ${ }^{2}$ Christopher L-H Huang ${ }^{2}$
}

\begin{abstract}
Atrial fibrillation, the commonest cardiac arrhythmia, predisposes to thrombus formation and consequently increases risk of ischaemic stroke. Recent years have seen approval of a number of novel oral anticoagulants. Nevertheless, warfarin and aspirin remain the mainstays of therapy. It is widely appreciated that both these agents increase the likelihood of bleeding: there is a popular conception that this risk is greater with warfarin. In fact, well-managed warfarin therapy (INR 2-3) has little effect on bleeding risk and is twice as effective as aspirin at preventing stroke. Patients with atrial fibrillation and a further risk factor for stroke (CHA2DS2VASC $>0$ ) should therefore either receive warfarin or a novel oral agent. The remainder who are at the very lowest risk of stroke are better not prescribed antithrombotic therapy. For stroke prevention in atrial fibrillation; aspirin is rarely the right choice.
\end{abstract}

\section{INTRODUCTION}

Atrial fibrillation (AF) is the most common cardiac arrhythmia affecting more than one in 20 Europeans aged over $55 .{ }^{12} \mathrm{AF}$ results in turbulent flow within the atria, predisposing to thrombus formation. Patients live with the constant risk that a fragment of thrombus will dislodge, embolise and occlude a cerebral artery, causing an ischaemic stroke: AF thus markedly increases stroke risk. ${ }^{3}$ One might expect that restoring sinus rhythm would obviate the stroke risk posed by AF. Surprisingly, however, a large body of data from the seminal RACE and AFFIRM trials confirm that it does not, likely reflecting ongoing undetected episodes of arrhythmia. ${ }^{4}$ It is therefore important to deal with the associated risk of stroke directly. This applies to patients with paroxysmal, as well as permanent, AF and also to those with atrial flutter. ${ }^{6}$ While the majority of large trials have focused on patients with nonvalvular $\mathrm{AF}$, this finding appears to be applicable to the broader AF and flutter populations.

While occlusion devices designed to prevent thrombus from breaking off and entering the circulation have been developed and show a good degree of efficacy, doubts remain as to their safety: at present they are only considered for use in patients at very high risk in whom other approaches are contraindicated. ${ }^{78}$ For the majority of patients, the mainstays of therapy to reduce stroke risk are antithrombotic medications. The impact of these drugs in reducing stroke risk is well established. ${ }^{9-15}$

There are two broad categories of antithrombotic drugs: antiplatelet agents and anticoagulants. Antiplatelet agents interfere with platelet function thereby reducing the likelihood that they will aggregate into a thrombus. Two main types of antiplatelet drugs have been studied for use in AF: aspirin and thienopyridine drugs such as clopidogrel. Of these, aspirin interferes with prostaglandin synthesis while the thienopyridines block binding of ADP to the platelet surface. Anticoagulant drugs, on the other hand, interfere with the clotting cascade, ultimately reducing the rate of fibrin clot formation. Until recently the only commonly used oral anticoagulant was warfarin, which blocks the formation of vitamin K-dependent clotting factors. In recent years, three new oral anticoagulant agents, rivaroxaban, apixaban and dabigatran, which block the final common pathway in clot formation, have come into the market.

The benefits of antithrombotic drugs as regards stroke prevention must be balanced against their known association with haemorrhage. While the majority of bleeds in patients on these drugs are minor, a proportion are major and life threatening: the most worrying form of such bleeding is intracerebral haemorrhage (ICH). Naturally, both the decision to treat and the choice of agent prescribed depend on an assessment of relative benefits and risks. An exaggerated perception of the bleeding risk associated with warfarin has resulted in its widespread underuse. This is effectively illustrated by a recent report that almost a third of patients with $\mathrm{AF}$ and flutter in the Loire Valley region of France are undertreated on the basis of American College of Cardiology/American Heart Association/ European Society of Cardiology (ESC) guidelines. ${ }^{16}$ Consequently, patients who might gain a net benefit from therapy unnecessarily suffer potentially devastating, and even fatal, strokes.

With the recent publication of the RE-LY, ${ }^{17}$ ROCKET-AF $^{18}$ and ARISTOTLE ${ }^{19}$ trials, attention has understandably focused on the potential benefits of the new oral anticoagulants as compared with warfarin. These drugs will doubtless come to form a useful part of our armoury in the future. When it comes to delivering impact for the majority of patients today, however, there are two key issues to tackle. First, one must make an informed decision as to whether or not to prescribe an antithrombotic drug, mindful of the attendant benefits and risks of therapy. Second, one must choose between antiplatelet and anticoagulant classes. In most cases this means a choice between aspirin and warfarin.

\section{STROKE RISK}

A number of meta-analyses confirm that warfarin treatment to a target international normalised ratio (INR) of 2.5, and a tolerated therapeutic range (TTR) of 2.0-3.0, reduces stroke risk by around two-thirds among the broad pool of patients with 
non-valvular AF. ${ }^{6} 2021$ This corresponds to an annual absolute risk reduction in all strokes of around $2.7 \% .^{22}$ The reduction in risk of both disabling and non-disabling strokes is similar. Importantly, the results of the BAFTA study confirm that benefit is especially marked in patients aged over $75 .^{23}$

This said, a number of the studies considered within these meta-analyses have been the subject of perhaps justifiable criticism on three key grounds. ${ }^{24}$ First, patients enrolled in a clinical trial are likely to be more regularly followed up than would otherwise have been expected in a real world setting. This might be expected to result in improved INR control, increasing the proportion of time in the TTR, which likely substantially impacts on outcomes. Second, several studies included within these meta-analyses report higher absolute stroke risks than are observed in modern practice, which may mean they overestimate the benefits of anticoagulation. ${ }^{25}$ Third, only patients for whom optimal choice of antithrombotic therapy is regarded as uncertain would usually be enrolled in comparison studies. Given previous data on the relatively greater efficacy of warfarin than aspirin in preventing stroke, it would have been regarded as unethical to deny patients warfarin therapy when it was clearly indicated. This means the patients selected would have been at a relatively lower risk of stroke as
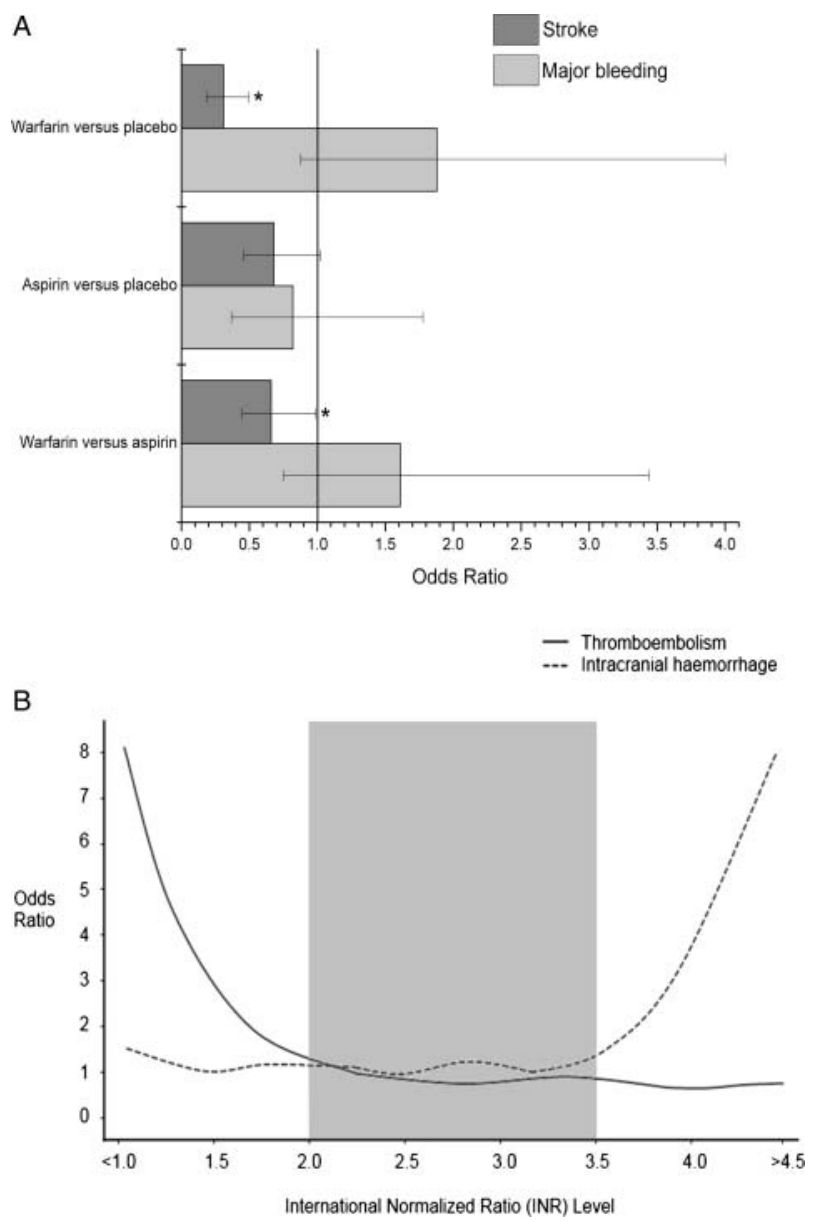

Figure 1 Impact of antithrombotic drugs on risk of stroke and major bleeding in patients with non-valvular atrial fibrillation. (A) Relative effects of aspirin and warfarin on risk of stroke and major bleeding. Lines indicate $95 \% \mathrm{Cls}$. Asterisks indicate differences which are significant at the $95 \%$ confidence level. Adapted from McNamara et al $(2004)^{32}$. (B) Impact of international normalised ratio on risk of stroke and intracranial haemorrhage. Illustration based on data from Singer et al $(2009)^{24}$. compared with the patient population as a whole: this was demonstrably the case in the BAFTA study. A recent retrospective meta-analysis of studies conducted in a real world setting provides clarity. Warfarin results in a two-thirds reduction in stroke risk in patients with at least one additional risk factor for stroke, such as those identified in the CHADS2 (congestive heart failure, hypertension, age over 75, diabetes, stroke history) scoring system, including the presence of congestive heart failure, hypertension, age over 75 or previous stroke. ${ }^{26}$

Importantly, data from the RE-LY, ROCKET-AF and ARISTOTLE trials respectively demonstrate that the new oral anticoagulants dabigatran, rivaroxaban and apixaban offer at least non-inferior stroke protection as compared with warfarin. ${ }^{17-19}$ The impact of the new oral anticoagulant agents on stroke risk, as well as points around trial design, are dealt with in detail elsewhere. ${ }^{27}$

Enthusiasm for the choice of aspirin for stroke prevention in AF is largely driven by the result of a single trial, SPAF- $1 .^{12}$ This reported a $42 \%$ reduction in overall stroke risk in patients randomised to aspirin as compared with those receiving placebo. Importantly, even in this study aspirin did not prevent clinically severe strokes. Furthermore, responses to aspirin were markedly heterogeneous and treatment appeared significantly less effective in the elderly. A meta-analysis of seven trials comparing the efficacy of aspirin and placebo in patients in AF who had additional risk factors for stroke reached a rather more modest conclusion: treatment with aspirin results in a non-significant one-fifth relative reduction in stroke risk. ${ }^{21}$ Interestingly, the magnitude of this relative risk reduction is similar to that seen when aspirin is given to patients with vascular disease: presumably a large proportion of those with AF also have vascular disease. ${ }^{28}$

Estimations of the efficacy of aspirin as compared with warfarin vary: a meta-analysis of six prevention trials suggests that it is around half as effective in preventing ischaemic strokes in patients with additional risk factors. ${ }^{6} 21$ A question remains as to whether aspirin is effective at preventing stroke in patients without additional risk factors (so-called lone $A F$ ): individual trials tend to include very few such patients. Meta-analyses focusing on this group suggest that aspirin again reduces the incidence of stroke in this group by around a fifth, though notably the CIs include the potential for harm. ${ }^{20} 21$ The role for aspirin in such patients thus remains unclear.

The question as to whether dual antiplatelet therapy with aspirin and a thienopyridine might offer an alternative anticoagulation strategy in at risk patients was comprehensively addressed by the ACTIVE-A and ACTIVE-W trials. ${ }^{29} 30$ In essence, combined treatment with aspirin and clopidogrel provides superior protection against stroke as compared with aspirin alone, but markedly inferior protection as compared with warfarin. Solely from the point of view of stroke risk, it therefore seems that combined treatment with aspirin and clopidogrel could be considered in patients at high risk of stroke who are not candidates for warfarin. Unfortunately, such a use of combination therapy to this end is obviated by the bleeding risk that it confers (see later). Finally, while combination treatment with aspirin and low-dose warfarin (eg, to an INR of 1.2-1.5) has previously been discussed, data from SPAF-III confirm that it is associated with a much higher stroke risk than is treatment with full-dose warfarin. ${ }^{31}$ It should therefore not be used for stroke prevention in AF.

Thus, there are good data demonstrating antithrombotics reduce stroke risk in patients with non-valvular $\mathrm{AF}$, with warfarin being significantly more effective that aspirin. This is illustrated in the meta-analysis data presented in figure $1 \mathrm{~A} .{ }^{32}$ 
Combination therapy with aspirin and a thienopyridine, or aspirin and low-dose warfarin, provides inferior protection against stroke and therefore should not be used for this indication.

\section{BLEEDING RISK}

A decade-old meta-analysis suggests that warfarin therapy carries a greater risk of major bleeding than does treatment with aspirin, with an absolute risk of 2.2 as compared with 1.3 events/100 patient years. ${ }^{6}$ Given the small magnitude of this difference, factors in trial design that may have influenced this conclusion require consideration. Based on prescribing behaviour at the time, one would expect that a patient assessed as being at a high risk of bleeding would be more likely to be treated with no medication than with aspirin, and more likely to be treated with aspirin than with warfarin. This selection bias might result in the average bleeding risk of a patient in the aspirin group being artificially low. The same argument could be made of the warfarin group. It is unclear how these factors might have played off against each other in these older trials.

More recent data from the BAFTA study suggest that rates of major bleeding in cohorts of elderly patients treated with warfarin or with aspirin are essentially indistinguishable. ${ }^{23}$ Thus, warfarin therapy resulted in an absolute risk of 1.4 extracranial bleeds/100 patient years as compared with 1.6 events with aspirin. One must interpret this striking finding with caution for three key reasons, however. ${ }^{33}$ First, four-fifths of patients enrolled in the BAFTA study were already taking warfarin or aspirin and thus had already established a track record of living safely on therapy. The same would not be true for a new patient for whom a therapy needs to be chosen. Second, the study did not allow combination therapy with warfarin and an antiplatelet agent. In real world clinical practice some patients will inevitably be on both types of medication. Third, as with other clinical trials, one might expect TTR to be greater than it would be in a real world setting. These issues are largely settled by a recently published real world study based on patient registry data: bleeding risk with well-controlled warfarin therapy was the same as that with aspirin. ${ }^{32}{ }^{34}$ It is thus clear that wellmanaged warfarin therapy results in little, if any, more bleeding that does treatment with aspirin, especially among older patients. $^{23} 34$

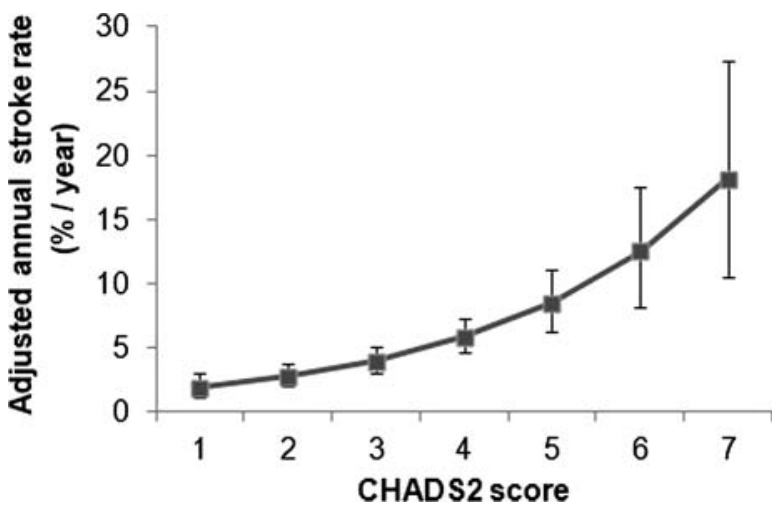

Figure 2 Variation in stroke risk with CHADS2 score. Bars indicate 95\% Cls. Stroke rates are adjusted using a multivariate model to remove the effect of aspirin usage. Note that stroke rates are declining and hence individual values may not reflect the magnitude of stroke risk today. Adapted from Gage et al (2001). ${ }^{35}$
It is important to note that, the comparisons highlighted are between low-dose $(75-100 \mathrm{mg})$ aspirin and well-managed warfarin therapy in which the patient has an INR that is within the TTR of 2.0-3.0 for the majority of the time. Bleeding risk is markedly greater in patients who are over-anticoagulated, that is, with an INR of greater than 3.0 (Singer et al 2009 ${ }^{24}$ ) (figure 1B). Stroke risk is effectively independent of INR at INRs of greater that 2.0: it is notable that many strokes among those on warfarin occur in patients in whom the INR is subtherapeutic. ${ }^{22} 35$ Well-executed clinical trials report TTRs in the region of 58\%$66 \%$ (Agarwal et al $2012^{10}$ ). There is a broad consensus that a TTR of at least $60 \%$ is both necessary and realistic: indeed, a TTR of less than $60 \%$ may entirely negate the benefit of warfarin therapy. ${ }^{28}$

Of course, it is sensible to think carefully about each patient's individualised bleeding risk, both prior to starting treatment and then on an ongoing basis. Various risk scoring systems such as HASBLED (hypertension, abnormal renal or liver function, stroke history, bleeding history, labile INR, elderly, ie, age over 65 , drug or alcohol use) have been proposed to identify patients in whom bleeding risk is of particular concern. ${ }^{36}{ }^{37}$ It should be noted, however, that the predictive accuracy of these scoring systems is relatively poor (with c-statistics in the range of 0.6).

As regards bleeding risk in patients receiving combination therapies, data from the ACTIVE-A and ACTIVE-W trials clearly show that dual antiplatelet therapy is associated with a similar bleeding risk as warfarin. ${ }^{29} 30$ Furthermore, the results of the SPAF-III trial demonstrate that treatment with aspirin and low-dose warfarin is associated with a markedly higher bleeding risk than full-dose warfarin alone. ${ }^{31}$ Concern over bleeding is therefore not a viable reason for choosing such therapies over warfarin.

Finally, it is notable that treatment with the new oral anticoagulant agents appears to be associated with a lower risk of ICH than warfarin. ${ }^{15-17}$ It should be borne in mind, however, that use of dabigatran is associated with a small, but statistically significant, increase in risk of myocardial infarction. ${ }^{38}$

\section{STRIKING A BALANCE}

The benefit of antithrombotic drugs, especially anticoagulants, in stroke prevention is clear. If these drugs were risk-free, all patients would be treated. The reality is that a decision to treat requires that the benefits of therapy as regards reduction in stroke risk must be weighed against the elevated risk of bleeding, and especially $\mathrm{ICH}$, they bring. Risk scoring systems which award points based on the presence of particular risk factors are useful for assessing a patient's stroke risk and thus informing a view on balance of risk between stroke and bleeding. Of these, the best validated is the CHADS2 scoring system mentioned earlier: annual stroke risk increases steeply with score (figure 2).

Modelling studies have calculated the net clinical benefit of warfarin therapy based on stroke risk as assessed using the CHADS2 system. Of these, the most informative has been the ATRIA study. ${ }^{24}$ Drawing on an extensive data set obtained in a real world setting over 6 years, it computed the difference between the annual rate of strokes prevented by warfarin and the annual rate of $\mathrm{ICH}$ due to warfarin. ICH risk was multiplied by a weighting factor of 1.5 , though notably the study's findings remained unchanged when this weighting factor was varied between 1.0 and 2.0. Extracranial haemorrhages, most of which do not pose a significant danger, were excluded.

ATRIA found net harm in patients treated with warfarin with a CHADS2 score of 0 , that is, in those without additional risk factors for stroke. The benefit of warfarin was equivocal at a 
CHADS2 score of 1 . Warfarin therapy resulted in a statistically significant net clinical benefit at CHADS2 scores of 2 or greater, with a marked increase in benefit with increasing score. It should be noted that the decision to anticoagulate in this study was made by the treating physicians who presumably concluded that patients were relatively safe candidates for anticoagulation, thus resulting in a potential underestimate of bleeding risk. Furthermore, half the control group were on aspirin, meaning that this study might underestimate the true benefit of warfarin as regards stroke reduction.

The conclusions of the ATRIA study are supported by those of the more recent Danish National Patient Registry Study. ${ }^{39}$ This reported a neutral impact or net benefit from warfarin at CHADS2 scores of 1 or greater, and even among patients with a CHADS2 of 0 if one additional risk factor included in the new CHA2DS2-VASc scoring system (atherosclerotic vascular disease,

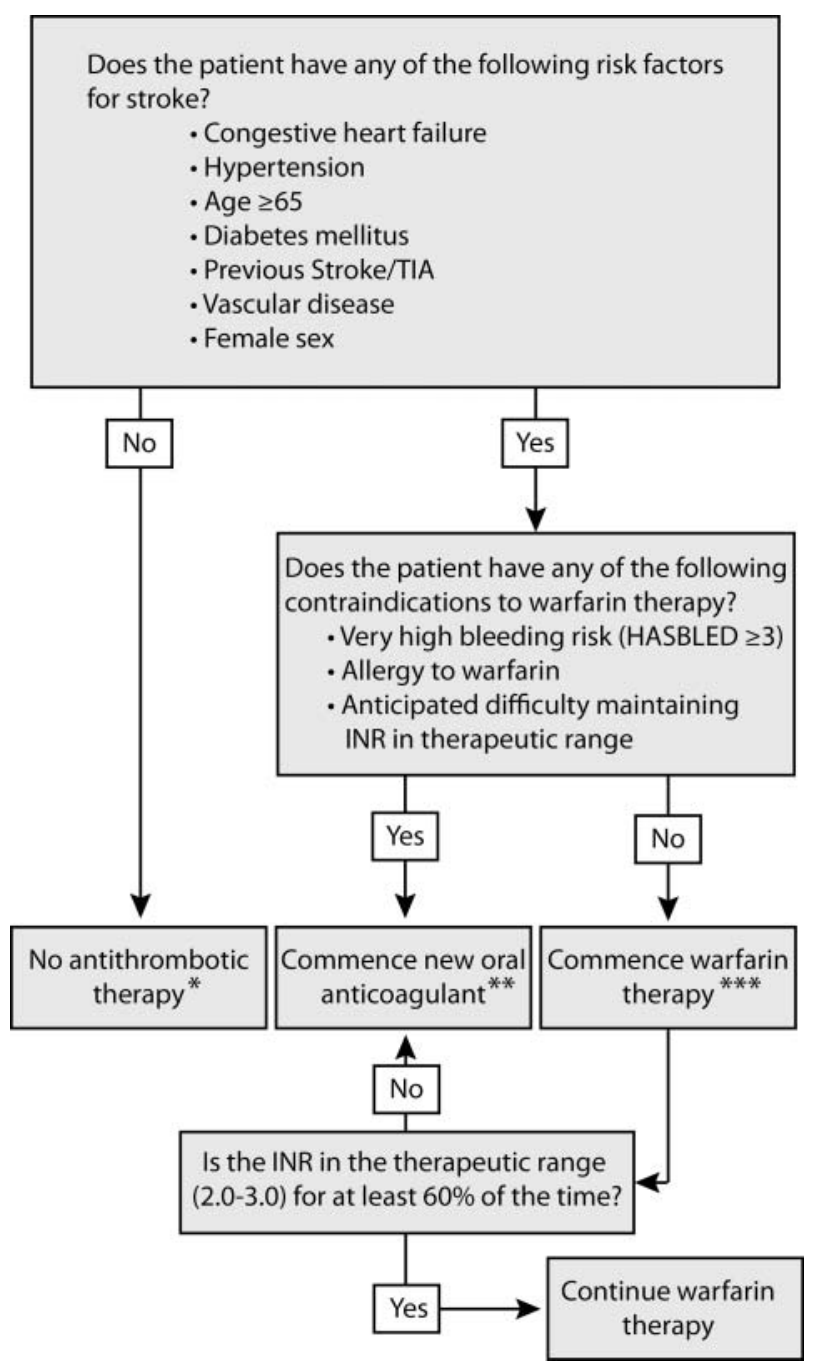

Figure 3 Algorithm for choosing antithrombotic therapy for stroke prevention in patients with non-valvular atrial fibrillation (AF). Recommendations based on 2010 European Society of Cardiology guidelines-see Camm et al (2010). ${ }^{28}$ * Aspirin may be considered in these patients, as well as in those with one risk factor for stroke, though it is not the preferred choice. ${ }^{* *}$ While these guidelines were published prior to the formal approval of the new oral anticoagulant agents for stroke prevention in AF, they nevertheless include mention of dabigatran for this indication. ${ }^{* * *}$ Or proceed directly to a new oral anticoagulant. INR, international normalised ratio; TIA, transient ischaemic attack. age between 65 and 74 or female sex, with additional consideration for age over 75) was present. Notably, there was no category of patients for whom aspirin therapy conferred a net clinical benefit.

Questions such as the applicability of these findings in the elderly often arise. It is notable that almost half of the patients enrolled in the ATRIA study were aged over 75. Interestingly, the net benefit of warfarin increased with patient age, being at a maximum among those aged 85 and over. This finding is consistent with that of the earlier BAFTA study, which reported substantial net benefit from warfarin among a cohort with a mean age of 82 . In the past, a fear over the potentially increased bleeding risk conferred by frequent falls has been cited as a reason to avoid warfarin in older patients. Not only does the ATRIA study argue against this view but outputs from a separate decision analysis model suggest that an AF patient would need to fall more than 295 times each year for the risk of ICH to outweigh potential stroke risk reduction by anticoagulation with warfarin. $^{40}$

The weight of evidence indicates that combination therapy with aspirin and low-dose warfarin should not be used, both because it does not offer effective protection against stroke and because its use is associated with an unacceptably high risk of ICH. ${ }^{31}$ Given that the ACTIVE-A and ACTIVE-W trials demonstrated that dual antiplatelet therapy provides inferior protection against stroke, yet results in the same bleeding risk as warfarin, ${ }^{29} 30$ a combination of aspirin and a thienopyridine should not be used for this indication. Patients at a high risk of stroke who cannot take warfarin would likely better be treated with one of the new oral anticoagulant agents. Dual antiplatelet therapy could only be justified among patients at a high risk of stroke who cannot take any such anticoagulants for reasons other than bleeding risk. This group is so small as to be virtually non-existent. There are therefore essentially no patients in whom antiplatelet therapy might be indicated for stroke prevention.

\section{CONCLUSIONS}

Among the broad pool of patients with AF, well-managed warfarin is far more effective than aspirin at preventing stroke. This makes physiological sense: warfarin is generally used to prevent thrombus formation in veins, whereas aspirin is used to prevent thrombus formation in arteries. While blood in the left atrial appendage, where stroke-causing thrombi tend to form in AF, is arterial, its haemodynamic characteristics are more in common with those of venous blood. At the same time, well-managed warfarin (with an INR in the TTR of 2.0-3.0 at least $60 \%$ of the time) increases $\mathrm{ICH}$ risk to a similar degree as aspirin. On this basis, the use of aspirin for stroke prevention in AF is difficult to justify. In the elderly these assertions not only hold, but are truer still.

For the individual patient, stroke risk scoring systems have an important place in guiding therapy. In essence, their role is to distinguish between two groups of patients: the minority who are at a very low stroke risk and the majority who are at a relatively higher risk. In the first group, defined by a CHA2DS2-VASc score of zero, the bleeding risk associated with anticoagulant therapy probably cannot be justified. In the second group, it clearly can. This is reflected in the preferred therapies suggested in current ESC guidelines, ${ }^{28}$ an adapted algorithm of which is presented in figure 3. Notably these guidelines recommend the continuation of anticoagulant therapy even following ablation procedures. 
The guidelines accommodate an important caveat as regards the use of warfarin. Its many drug-drug and drug-food interactions, as well as the frequency of monitoring required, will make achieving an adequate TTR an unrealistic challenge in some patients. Furthermore, it may present an undue risk of

\section{Main messages}

- Warfarin is superior to aspirin at preventing stroke in patients with atrial fibrillation.

- Well-managed warfarin therapy results in a similar risk of major bleeding as treatment with aspirin.

- While the majority of patients with atrial fibrillation should be anticoagulated, those with no other risk factors for stroke (CHA2DS2-VASc score of zero) are best not prescribed any such therapy.

- As compared with warfarin, the new oral anticoagulants have similar impact on stroke risk yet result in a lower bleeding risk.

- Aspirin is rarely the right choice of antithrombotic therapy for managing stroke risk in atrial fibrillation.

\section{Current research questions}

- What is the optimal management for patients with paroxysmal atrial fibrillation?

- Do the balance of clinical and health economic considerations point to a decreasing future role for warfarin, and a growing role for the new oral anticoagulant agents?

- Is there any place for aspirin in managing stroke risk in atrial fibrillation?

\section{Key references}

- Camm AJ, Kirchhof P, Lip GYH, et al. Guidelines for the management of atrial fibrillation. Europace 2010;12:1360420.

- Mant J, Hobbs FD, Fletcher K, et al. Warfarin versus aspirin for stroke prevention in an elderly community population with atrial fibrillation (the Birmingham Atrial Fibrillation Treatment of the Aged Study, BAFTA): a randomised controlled trial. The Lancet 2007;370:493-503.

- Singer DE, Chang Y, Fang MC, et al. The net clinical benefit of warfarin anticoagulation in atrial fibrillation. Ann Intern Med 2009;151:297-305.

- Friberg L, Rosenqvist M, Lip GYH. Evaluation of risk stratification schemes for ischaemic stroke and bleeding in 182678 patients with atrial fibrillation: the Swedish Atrial Fibrillation cohort study. Eur Heart J 2012;33:1500.

- Man-Son-Hing M, Nichol G, Lau A, et al. Choosing antithrombotic therapy for elderly patients with atrial fibrillation who are at risk for falls. Arch Intern Med 1999;159:677. bleeding in those patients with the very highest bleeding risk scores (HASBLED score of $\geq 3$ ). Such patients will still benefit from anticoagulation: antiplatelet therapy with aspirin is not an appropriate choice in this setting. Given that the new oral anticoagulants appear to present a lower bleeding risk than warfarin, set against at least equivalent protection against stroke, one of these agents would seem a sensible next choice. While the current ESC guidelines for antithrombotic therapy in AF were published prior to the approval of these new agents, they nevertheless include a place for the use of dabigatran. ${ }^{28}$ Since their publication, dabigatran and rivaroxaban have been approved for stroke prevention in AF in both the USA and Europe.

In the future, the new oral anticoagulant drugs may come to replace warfarin as the first line anticoagulant therapy in patients with AF. Until the current scientific, clinical and health economic arguments surrounding the use of these agents are resolved, however, warfarin will remain the first choice in the majority of patients. In any case, aspirin is rarely the right choice of antithrombotic therapy for stroke prevention in AF.

\section{MULTIPLE CHOICE QUESTIONS}

1. Regarding the new oral anticoagulants:

a. dabigatran is mentioned in the 2010 European Society of Cardiology guidelines as being potentially useful for stroke prevention in atrial fibrillation (T)

b. these agents are generally associated with a greater risk of bleeding than is well-managed warfarin therapy $(\mathrm{F})$

c. they may be used as first-line agents $(T)$

d. dabigatran has been associated with a small increase in risk of myocardial infarction $(\mathrm{T})$

e. these agents work by blocking the production of vitamin-K dependent clotting factors $(\mathrm{F})$

2. Regarding warfarin:

a. warfarin therapy can safely be discontinued following an atrial fibrillation ablation procedure $(\mathrm{F})$

b. well-managed therapy is reflected by a time in therapeutic range (TTR) of greater than $60 \%(\mathrm{~T})$

c. the benefits of warfarin therapy as regards stroke prevention are especially marked in elderly patients $(\mathrm{T})$

d. a patient warfarinised to an international normalised ratio (INR) of 3.0 is at a markedly greater risk of intracerebral haemorrhage as compared to a patient with an INR of $1.0(\mathrm{~F})$

e. well-managed warfarin therapy is associated with a broadly similar bleeding risk as compared aspirin therapy (T)

3. Regarding aspirin:

a. enthusiasm for aspirin use for stroke prevention in atrial fibrillation was driven by the results of a single trial, the results of which have now been superseded (T)

b. aspirin may be safely combined with low-dose warfarin (F)

c. aspirin is an antiplatelet drug (T)

d. combination therapy with aspirin and clopidogrel may be indicated for stroke prevention in some patients with atrial fibrillation $(\mathrm{F})$

e. aspirin therapy is useful in patients in whom warfarin cannot be used $(\mathrm{F})$

4. Regarding risk scoring systems used in guiding treatment decisions in patients with atrial fibrillation:

a. the CHADS2 scoring system awards a point if a patient is aged over $65(\mathrm{~F})$

b. the CHA2DS2-VASc scoring system awards a point for male sex $(\mathrm{F})$ 
c. a patient with a HASBLED score of 4 is considered to be at a very high risk of bleeding $(T)$

d. the predictive power of the HASBLED scoring system is relatively poor $(\mathrm{T})$

e. together a patient's CHA2DS2-VASc and HASBLED scores are sufficient to reach a decision as to which therapy to choose $(\mathrm{F})$

5. Given the clinical contexts, the following therapeutic choices are suitable:

a. a 30 year old man with atria fibrillation and no other relevant risk factors - no therapy $(\mathrm{T})$

b. a 55 year old woman with hypertension and no contra-indications to warfarin therapy - dabigatran $(\mathrm{T})$

c. a 75 year old man with congestive heart failure, hypertension and a HASBLED score of 4 - warfarin $(F)$

d. a 75 year old woman with peripheral vascular disease and no contra-indications to warfarin therapy - warfarin $(\mathrm{T})$

e. an 80 year old woman receiving warfarin therapy with a CHADS2-VASc score of 3 an a time in therapeutic range (TTR) of $65 \%$ - warfarin $(\mathrm{T})$

Acknowledgements We thank Dr Victoria Jones for her help in figure preparation. We gratefully acknowledge support from the British Heart Foundation, Wellcome Trust, Medical Research Council, Biotechnology and Biological Sciences Research Council, Stanley-Elmore Fund, Gonville and Caius College, the University of Cambridge's Translational Medicine and Therapeutics Program and the National Institutes for Health Research.

Contributors All authors provided written sections for this review.

Competing interests None.

Provenance and peer review Not commissioned; externally peer reviewed.

Correction notice This article has been updated since it was published Online First. The multiple choice questions have been corrected.

Open Access This is an Open Access article distributed in accordance with the Creative Commons Attribution Non Commercial (CC BY-NC 3.0) license, which permits others to distribute, remix, adapt, build upon this work non-commercially, and license their derivative works on different terms, provided the original work is properly cited and the use is non-commercial. See: http://creativecommons.org/licenses/by-nc/3.0/

\section{REFERENCES}

1 Heeringa J, Van Der Kuip DAM, Hofman A, et al. Prevalence, incidence and lifetime risk of atrial fibrillation: the Rotterdam study. Eur Heart J 2006:27:949-53.

2 Wyndham CRC. Atrial fibrillation: the most common arrhythmia. Tex Heart Inst J 2000;27:257

3 Stewart S, Hart CL, Hole DJ, et al. A population-based study of the long-term risks associated with atrial fibrillation: 20-year follow-up of the Renfrew/Paisley study. Am J Med 2002;113:359-64.

4 Wyse DG, Waldo AL, DiMarco JP, et al. A comparison of rate control and rhythm control in patients with atrial fibrillation. N Engl J Med 2002;347:1825.

5 Van Gelder IC, Hagens VE, Bosker HA, et al. A comparison of rate control and rhythm control in patients with recurrent persistent atrial fibrillation. N Engl J Med 2002;347:1834-40

6 van Walraven $C$, Hart RG, Singer DE, et al. Oral anticoagulants vs aspirin in nonvalvular atrial fibrillation. JAMA 2002;288:2441-8.

7 Holmes DR, Reddy VY, Turi ZG, et al. Percutaneous closure of the left atrial appendage versus warfarin therapy for prevention of stroke in patients with atria fibrillation: a randomised non-inferiority trial. Lancet 2009;374:534-42.

8 Viles-Gonzalez JF, Kar S, Douglas P, et al. The clinical impact of incomplete left atrial appendage closure with the Watchman Device in patients with atrial fibrillation: a PROTECT AF (Percutaneous closure of the left atrial appendage versus warfarin therapy for prevention of stroke in patients with atrial fibrillation) substudy. J Am Coll Cardiol 2012:59:923-9.

9 Warfarin versus aspirin for prevention of thromboembolism in atrial fibrillation: stroke prevention in Atrial Fibrillation II Study. Lancet 1994:343:687-91.

10 Agarwal S, Hachamovitch R, Menon V. Current trial-associated outcomes with warfarin in prevention of stroke in patients with nonvalvular atrial fibrillation: a meta-analysis. Arch Intern Med 2012;172:623.

11 The effect of low-dose warfarin on the risk of stroke in patients with nonrheumatic atrial fibrillation. The Boston Area Anticoagulation Trial for Atrial Fibrillation Investigators. N Engl J Med 1990;323:1505-11.
12 McBride R. Stroke prevention in atrial fibrillation study: final results. Circulation 1991;84:527-39.

13 Petersen P, Godtfredsen J, Boysen G, et al. Placebo-controlled, randomised trial of warfarin and aspirin for prevention of thromboembolic complications in chronic atrial fibrillation: the Copenhagen AFASAK study. Lancet 1989;333:175-9.

14 Ezekowitz MD, Bridgers SL, James KE, et al. Warfarin in the prevention of stroke associated with nonrheumatic atrial fibrillation. N Engl J Med 1992;327: 1406-12.

15 Connolly SJ, Laupacis A, Gent M, et al. Canadian atrial fibrillation anticoaguiation (CAFA) study. J Am Coll Cardiol 1991:18:349-55.

16 Gorin L, Fauchier L, Nonin E, et al. Prognosis and guideline-adherent antithrombotic treatment in patients with atrial fibrillation and atrial flutter. Chest 2011;140:911-17.

17 Connolly SJ, Ezekowitz MD, Yusuf S, et al. Dabigatran versus warfarin in patients with atrial fibrillation. N Engl J Med 2009:361:1139-51.

18 Patel MR, Mahaffey KW, Garg J, et al. Rivaroxaban versus warfarin in nonvalvular atrial fibrillation. N Engl J Med 2011:365:883-91.

19 Granger CB, Alexander JH, McMurray JJV, et al. Apixaban versus warfarin in patients with atrial fibrillation. N Engl J Med 2011;365:981-92.

20 Cooper NJ, Sutton AJ, Lu G, et al. Mixed comparison of stroke prevention treatments in individuals with nonrheumatic atrial fibrillation. Arch Intern Med 2006;166:1269.

21 Hart RG, Pearce LA, Aguilar MI. Meta-analysis: antithrombotic therapy to prevent stroke in patients who have nonvalvular atrial fibrillation. Ann Intern Med 2007;146:857-67.

22 Lip GYH, Nieuwlaat R, Pisters R, et al. Refining clinical risk stratification for predicting stroke and thromboembolism in atrial fibrillation using a novel risk factor-based approach. Chest 2010;137:263-72.

23 Mant J, Hobbs FD, Fletcher K, et al. Warfarin versus aspirin for stroke prevention in an elderly community population with atrial fibrillation (the Birmingham Atrial Fibrillation Treatment of the Aged Study, BAFTA): a randomised controlled trial. Lancet 2007:370:493-503.

24 Singer $\mathrm{DE}$, Chang $\mathrm{Y}$, Fang $\mathrm{MC}$, et al. The net clinical benefit of warfarin anticoagulation in atrial fibrillation. Ann Intern Med 2009;151:297-305.

25 Go AS, Hylek EM, Chang Y, et al. Anticoagulation therapy for stroke prevention in atrial fibrillation. JAMA 2003:290:2685-92.

26 Ogilvie IM, Welner SA, Cowell W, et al. Ischaemic stroke and bleeding rates in'real-world'atrial fibrillation patients. Thromb Haemost 2011:106:34.

27 Spinler SA, Shafir V. New oral anticoagulants for atrial fibrillation. Circulation 2012;126:133-7.

28 Camm AJ, Kirchhof $\mathrm{P}$, Lip GYH, et al. Guidelines for the management of atrial fibrillation. Europace 2010;12:1360-420

29 Site HG. Clopidogrel plus aspirin versus oral anticoagulation for atrial fibrillation in the Atrial fibrillation Clopidogrel Trial with Irbesartan for prevention of Vascular Events (ACTIVE W): a randomised controlled trial. Lancet 2006:367:1903-12.

30 Connolly SJ, Pogue J, Hart RG, et al. Effect of clopidogrel added to aspirin in patients with atrial fibrillation. N Engl J Med 2009:360:2066-78.

31 Blackshear JL, Baker VS, Rubino F, et al. Adjusted-dose warfarin versus low-intensity, fixed-dose warfarin plus aspirin for high-risk patients with atrial fibrillation: stroke prevention in Atrial Fibrillation III randomised clinical trial. Lancet 1996;348:633-8

32 McNamara RL, Tamariz LJ, Segal JB, et al. Management of atrial fibrillation: review of the evidence for the role of pharmacologic therapy, electrical cardioversion, and echocardiography. Ann Intern Med 2003:139:1018-33.

33 Garcia D, Hylek E. Stroke prevention in elderly patients with atrial fibrillation. Lancet 2007;370:460-1.

34 Friberg L, Rosenqvist M, Lip GYH. Evaluation of risk stratification schemes for ischaemic stroke and bleeding in 182678 patients with atrial fibrillation: the Swedish Atrial Fibrillation cohort study. Eur Heart J 2012;33:1500-10.

35 Gage BF, Waterman AD, Shannon W, et al. Validation of clinical classification schemes for predicting stroke. JAMA 2001;285:2864-70.

36 Pisters $R$, Lane DA, Nieuwlaat $R$, et al. A novel user-friendly score (HAS-BLED) to assess 1-year risk of major bleeding in patients with atrial fibrillation: the Euro Heart Survey. Chest 2010;138:1093-100

37 Lip GYH, Frison L, Halperin JL, et al. Comparative validation of a novel risk score for predicting bleeding risk in anticoagulated patients with atrial fibrillation: the HAS-BLED (Hypertension, Abnormal Renal/Liver Function, Stroke, Bleeding History or Predisposition, Labile INR, Elderly, Drugs/Alcohol Concomitantly) score. J Am Coll Cardiol 2011:57:173-80.

38 Uchino K, Hernandez AV. Dabigatran association with higher risk of acute coronary events: meta-analysis of noninferiority randomized controlled trials. Arch Intern Med 2012:172:397-402

39 Olesen JB, Lip GYH, Lindhardsen J, et al. Risks of thromboembolism and bleeding with thromboprophylaxis in patients with atrial fibrillation: a net clinical benefit analysis using a'real world'nationwide cohort study. Thromb Haemost 2011:106:739.

40 Man-Son-Hing M, Nichol G, Lau A, et al. Choosing antithrombotic therapy for elderly patients with atrial fibrillation who are at risk for falls. Arch Intern Med 1999;159:677. 